\title{
Effectiveness of Training Programs in Reducing Falls in Older Adults: Systematic Review
}

\author{
Alexandre S Silva ${ }^{1}$, José Jonas de Oliveira ${ }^{1 *}$, Geovani S Andrade ${ }^{1}$, Jenifer CS Nascimento ${ }^{1}$, Anna \\ Gabriela SV Ribeiro ${ }^{2}$, Carolina GR Barbosa ${ }^{2}$, Jasiele AO Silva ${ }^{1}$ and Adriana Pertille ${ }^{2}$ \\ ${ }^{1}$ Department of Physical Education, University Centre of Itajubá (FEPI), Brazil \\ ${ }^{2}$ Post-graduate Program in Human Movement Sciences - Methodist, University of Piracicaba (UNIMEP), Brazil
}

*Corresponding author: José Jonas de Oliveira, Department of Physical Education, University Centre of Itajubá (FEPI), Brazil

\section{ARTICLE INFO}

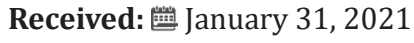

Published: March 12, 2021

Citation: Alexandre S S, José Jonas de Oliveira, Geovani S A, Jenifer CS Nascimento, Anna Gabriela SV Ribeiro, et al., Effectiveness of Training Programs in Reducing Falls in Older Adults: Systematic Review. Biomed J Sci \& Tech Res 34(3)2021. BJSTR. MS.ID.005564.

Keywords: Risk of Falling; Older Adults; Training Program; Balance

\section{ABSTRACT}

Background: With ageing, older adults are faced with decreasing strength and balance and increasing risk for falls. A fall followed by fracture is a factor in mortality and decreased quality of life in older people. However, few systematic review studies have analyzed the effects of training programs in reducing falls in older people.

Aim: This systematic review study aimed to analyze the effects of physical training programs on the risk of falls in older people.

Methods: We used the PRISMA guidelines in conducting the systematic review. The search for eligible studies was performed using four electronic databases (PubMed, BVS Regional Portal, British Library, and Library of Congress).

Results: The results showed a decrease in the risk of falling in 21 studies. Only four studies did not show a reduction in the risk of falling after a training program.

Conclusion: Combined or multicomponent training programs, as well as isolated strength training, could decrease the risk of falling in older people.

\section{Core Tip}

Regular and systematic physical exercises can contribute in a comprehensive way to the healthy ageing process by helping increase muscle strength, bone mineral density (hip and spine), and static and dynamic balance, ultimately decreasing the risk of falling. However, controversial results have been observed, with some studies indicating no improvement, especially in the risk of falls in older adults. Thus, the characteristics of the different physical exercise programs must be investigated. Research should discern which types of exercise reduce the risk of falling in the older adults. Thus, this systematic review study aimed to analyses the effects of physical training programs on the risk of falls in older adults.

\section{Introduction}

The global projection is that by 2050, the number of older people will increase exponentially, surpassing the number of children under 14 years old [1]. As people age, changes in muscle and bone mass can be observed, as well as reduction in the capacity to produce strength, which impacts the activities of daily living. Thus, regular physical activities can delay marked physiological losses. Falls followed by fractures are recurrent among older adults, attributable to muscle instability and bone fragility [2,3]. Changes in the sensory and motor systems also become present, and these cause postural instability, which can increase the risk of falling. Falls in older people represent a major public health problem, associated with a worsening quality of life and increased mortality [4]. In Brazil, about one-third of older adults experience at least one fall per year, and the more advanced the age, the greater the risk. Women are affected in greater proportion, as they are more sensitive to changes inherent to ageing, such as hormonal changes, 
which increase the incidence of sarcopenia and osteoporosis and make them more susceptible to falling [5].

Another factor associated with the risk of falling is the level of dependency of older adults, which can reach up to 14 times that of those with a high level of independence [6]. Regular and systematic physical exercises can contribute in a comprehensive way to the healthy ageing process by helping increase muscle strength, bone mineral density (hip and spine), and static and dynamic balance, ultimately decreasing the risk of falling $[4,7,8]$.

However, controversial results have been observed, with some studies indicating no improvement, especially in the risk of falls in older adults [9-12]. Thus, the characteristics of the different physical exercise programs must be investigated. Research should discern which types of exercise reduce the risk of falling in the older adults, for example, isolated (strength, aerobic, and balance) or combined (balance/strength, aerobic/balance/strength, aerobic/ balance, and aerobic/strength). Thus, this systematic review study aimed to analyses the effects of physical training programs on the risk of falls in older adults.

\section{Materials and Methods}

\section{Search Strategy}

For the review study, we adhered to the guidelines for systematic reviews (PRISMA) Moher, et al. [13]. The search for eligible studies was carried out using four electronic databases (PubMed, BVS Regional Portal, British Library, and Library of Congress) until October 2019, without language restrictions. The terms used for the search were as follows: the search strategy combined terms covering the topics of population: (resistance training OR strength training) AND (balance training) AND (older OR elderly OR older adults) AND (risk of falling). The search was carried out using combinations of the following terms linked with Boolean operators "AND" (inter-group Boolean operator) and "OR" (intra-group Boolean operator). Duplicate studies were removed. Three independent researchers analyzed titles and abstracts and then selected the articles relevant to the research.

\section{Eligibility Criteria}

Articles that met the following inclusion criteria were selected:

(1) Original study;

(2) Providing a description of the training program;

(3) Having at least one of the following tests as a method of evaluation: Balance Evaluation System Test, Timed Up and Go Test, Six Minute Walk Test, Sit To Stand Test, Falls Efficacy Scale-
Internacional, Posturografia estática BioRescue, Functional Reach Test, and Berg Balance Scale;

(4) Randomised clinical trials;

(5) Published in peer-reviewed scientific journals; and

(6) Studies evaluating the effects of training programs on the risk of falls in older adults.

The exclusion criteria were as follows:

(1) Review articles;

(2) Abstracts of unpublished conference papers and studies; and

(3) No protocol for assessing balance or risk of falling.

According to the inclusion and exclusion criteria, 19 studies were excluded. The studies were evaluated in their entirety by two reviewers. Disagreements were decided by consensus, and when necessary, with the participation of a third reviewer. Eligible study references were also analyzed to identify other relevant studies.

\section{Data Extraction}

Independently, the same two reviewers extracted the following data from each selected study: name of the first author, year of publication, research title, age group (years), balance test, type of exercise used in the training program, weekly frequency of the training program, intensity and duration of the training program, training period in months, completion of the study, and periodicals in which the studies were published.

\section{Evaluation of Methodological Quality of Studies}

The quality evaluation of the eligible studies was carried out by two independent reviewers, with doubts resolved by consensus, and with the participation of a third reviewer when no consensus could be reached. The Physiotherapy Evidence Database (PEDro) scale was used to assess randomised clinical trials. The instrument consists of 11 items, the first of which is not computed in the final score. Thus, the scale has a score from 0 to 10 [14].

\section{Results}

\section{Study Selection}

From the electronic databases (PubMed, BVS Regional Portal, British Library, and Library of Congress), 17727 studies were found. After removing the duplicates, 17610 remained. After the selection based on titles and abstracts, 44 complete articles remained. Of these, 25 were selected and used in the present work, as shown in Figure 1. 


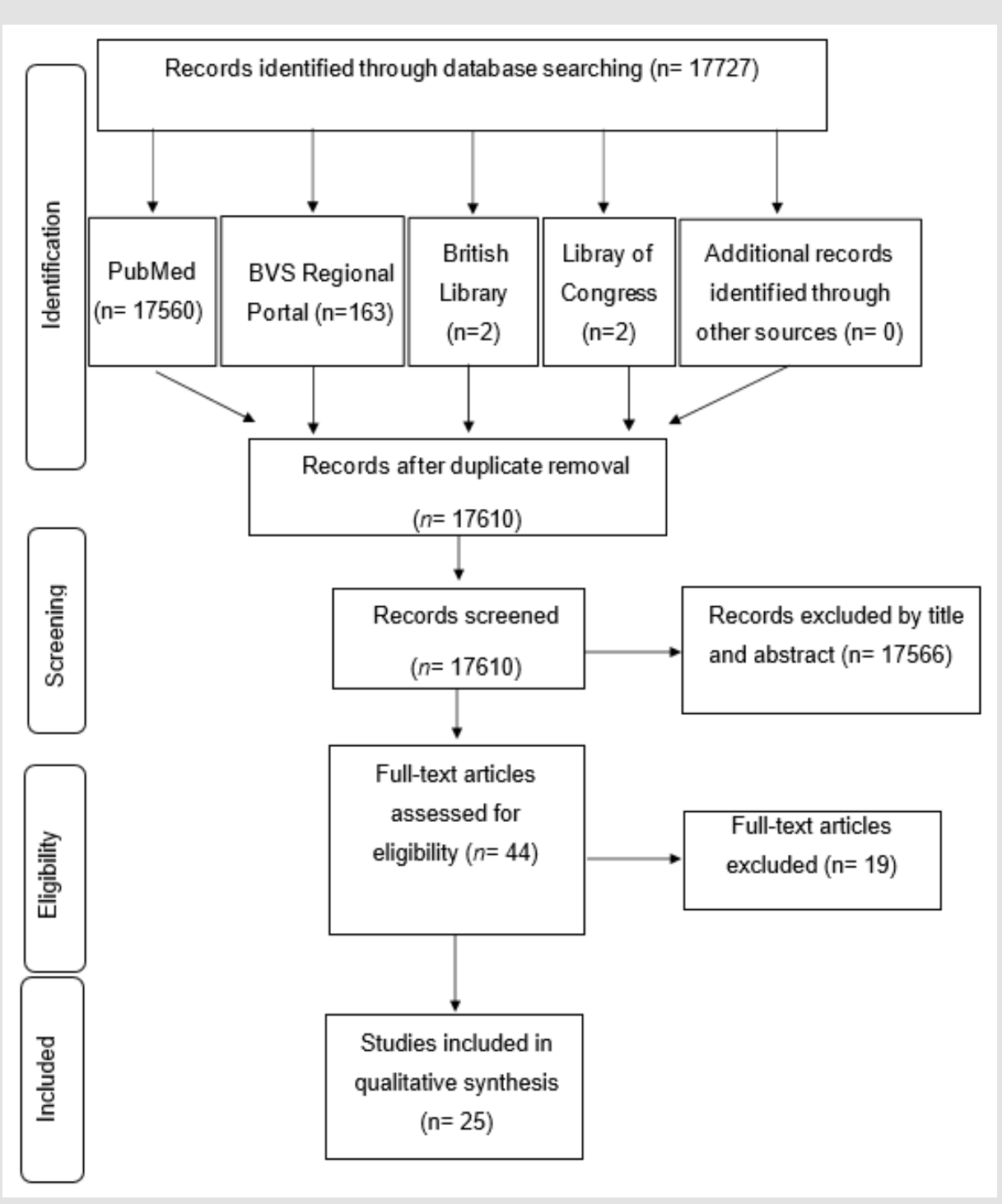

Figure 1: Organization of articles reviewing the effects of training programs on the risk of falls in the elderly. PRISMA 2009 flow diagram. Adapted from Moher, et al. [13].

\section{Study Characteristics}

Table 1 presents the general characteristics and results of studies that analyzed the effects of training programs on the risk of falls in older adults. The analyzed studies were published between 2008 and 2019, including a total of 2229 people. The studies were conducted in Australia [15], Brazil [4,10,16-18], Canada [19], China [20], Finland [21], Germany [22], Hungary [23], India [24], Iran [25], Japan [11], Portugal [7,26], Republic of Korea [27], Spain $[12,28]$, Switzerland [29], and the United States [9,30-33]. Eight studies were identified as using a combination of balance and strength exercises $[4,16,17,19,21,22,24,29]$; three studies using a combination of aerobic exercises, balance, and strength $[7,9,28]$; six studies using strength exercises [15,20,25,27,30,33]; one study using a combination of aerobic and strength exercises [10]; one study using aerobic exercises [12]; four studies using balance exercises [11,23,31,32]; and two studies using balance and strength exercises separately $[18,26]$. A reduction in the risk of falling was observed in 21 studies [4,7,15-33]. In four studies, training programs did not show a reduction in the risk of falling [9-12]. To evaluate the risk of falling in the older population, 16 studies used the Timed Up and Go Test [4,7,9,11,12,15-22,25,26,29]. Some studies opted for other tests to assess the risk of falling, such as the Balance Evaluation System Test [28], Six Minute Walk Test [30,33], Sit-to-Stand [10], Falls Efficacy Scale-Internacional [31], Posturografia estática BioRescue [27], Functional Reach Test [24], and Berg Balance Scale [23,32]. 
Table 1: Results of studies of training programs' effects $(n=25)$ on the risk of falls in older adults.

\begin{tabular}{|c|c|c|c|c|c|c|c|c|c|c|}
\hline \multirow[b]{2}{*}{ № } & \multirow[b]{2}{*}{ Author(s) } & \multirow[b]{2}{*}{ Title } & \multirow[b]{2}{*}{ Age (y) } & \multirow[b]{2}{*}{$\begin{array}{l}\text { Method of } \\
\text { Evaluation }\end{array}$} & \multicolumn{4}{|c|}{ Training Program (Protocol) } & \multirow[b]{2}{*}{$\begin{array}{c}\text { Conclusion } \\
\text { Regarding } \\
\text { Risk of Falls }\end{array}$} & \multirow[b]{2}{*}{ Journal } \\
\hline & & & & & $\begin{array}{l}\text { Type of } \\
\text { Exercise }\end{array}$ & $\begin{array}{l}\text { Weekly } \\
\text { Fre- } \\
\text { quency }\end{array}$ & $\begin{array}{c}\text { Intensity } \\
\text { and } \\
\text { Duration }\end{array}$ & $\begin{array}{c}\text { Period } \\
\text { in } \\
\text { Week }\end{array}$ & & \\
\hline 1 & $\begin{array}{c}\text { Teixeira et } \\
\text { al. [4] }\end{array}$ & $\begin{array}{l}\text { Progressive load } \\
\text { training for the } \\
\text { quadriceps muscle } \\
\text { associated with } \\
\text { proprioception } \\
\text { exercises for the } \\
\text { prevention of falls } \\
\text { in postmenopausal } \\
\text { women with } \\
\text { osteoporosis: } \\
\text { a randomized } \\
\text { controlled trial }\end{array}$ & $55-75$ & TUG & $\begin{array}{l}\text { Combination } \\
\text { of balance } \\
\text { and strength } \\
\text { exercises }\end{array}$ & - & $\begin{array}{l}\text { moderate- } \\
\text { to high- } \\
\text { intensity } \\
60 \text { min }\end{array}$ & 4,5 & $\begin{array}{l}\text { Reduced the } \\
\text { risk of falling }\end{array}$ & Osteoporos Int \\
\hline 2 & $\begin{array}{c}\text { Sousa et al. } \\
\text { [7] }\end{array}$ & $\begin{array}{l}\text { Combined exercise is } \\
\text { more effective than } \\
\text { aerobic exercise in } \\
\text { the improvement } \\
\text { of fall risk factors: } \\
\text { a randomized } \\
\text { controlled trial in } \\
\text { community-dwelling } \\
\text { older men }\end{array}$ & $65-79$ & TUG & $\begin{array}{l}\text { Combination } \\
\text { of aerobic } \\
\text { exercises and } \\
\text { balance }\end{array}$ & 3 & $\begin{array}{l}\text { moderate- } \\
\text { to high- } \\
\text { intensity } \\
60 \text { min }\end{array}$ & 8 & $\begin{array}{l}\text { Reduced the } \\
\text { risk of falling }\end{array}$ & $\begin{array}{c}\text { Clinical } \\
\text { Rehabilitation }\end{array}$ \\
\hline 3 & $\begin{array}{c}\text { Cancela et } \\
\text { al. [9] }\end{array}$ & $\begin{array}{c}\text { Effects of Three } \\
\text { Different Chair-Based } \\
\text { Exercise Programs on } \\
\text { People Older Than } 80 \\
\text { Years }\end{array}$ & $>80$ & TUG & $\begin{array}{c}\text { Aerobic } \\
\text { exercises, } \\
\text { Balance, and } \\
\text { Strength } \\
\text { exercises }\end{array}$ & 3 & $\begin{array}{l}\text { Low- to } \\
\text { moderate- } \\
\text { intensity } \\
60 \text { min }\end{array}$ & 3 & $\begin{array}{l}\text { Did not } \\
\text { reduce the } \\
\text { risk of falling }\end{array}$ & $\begin{array}{l}\text { Rejuvenation } \\
\text { Research }\end{array}$ \\
\hline 4 & $\begin{array}{c}\text { Ansai et al. } \\
{[10]}\end{array}$ & $\begin{array}{l}\text { Effects of two } \\
\text { physical exercise } \\
\text { protocols on physical } \\
\text { performance related } \\
\text { to falls in the oldest } \\
\text { old: A randomized } \\
\text { controlled trial }\end{array}$ & $>80$ & Sit-to- stand & $\begin{array}{l}\text { Combination } \\
\text { of aerobic } \\
\text { and strength } \\
\text { exercises }\end{array}$ & 3 & $\begin{array}{l}\text { moderate- } \\
\text { to high- } \\
\text { intensity } \\
60 \text { min }\end{array}$ & 4 & $\begin{array}{l}\text { Did not } \\
\text { reduce the } \\
\text { risk of falling }\end{array}$ & $\begin{array}{l}\text { Geriatrics } \\
\text { Gerontology }\end{array}$ \\
\hline 5 & $\begin{array}{l}\text { Hiyamizu } \\
\text { et al. [11] }\end{array}$ & $\begin{array}{l}\text { Effects of dual } \\
\text { task balance } \\
\text { training on dual } \\
\text { task performance } \\
\text { in elderly people: } \\
\text { a randomized } \\
\text { controlled trial }\end{array}$ & $>65$ & TUG & $\begin{array}{l}\text { Balance } \\
\text { exercises }\end{array}$ & 2 & $\begin{array}{l}\text { Low- to } \\
\text { moderate- } \\
\text { intensity } \\
60 \text { min }\end{array}$ & 12 & $\begin{array}{l}\text { Did not } \\
\text { reduce the } \\
\text { risk of falling }\end{array}$ & $\begin{array}{c}\text { Sage Journals } \\
\text { - Clinical } \\
\text { Rehabilitation }\end{array}$ \\
\hline 6 & $\begin{array}{c}\text { Varela et al. } \\
\text { [12] }\end{array}$ & $\begin{array}{l}\text { Effects of two } \\
\text { different intensities } \\
\text { of aerobic exercise } \\
\text { on elderly people } \\
\text { with mild cognitive } \\
\text { impairment: a } \\
\text { randomized pilot } \\
\text { study }\end{array}$ & $>79$ & TUG & $\begin{array}{c}\text { Aerobic } \\
\text { exercises }\end{array}$ & 3 & $\begin{array}{l}\text { Low- to } \\
\text { moderate- } \\
\text { intensity } \\
60 \text { min }\end{array}$ & 12 & $\begin{array}{l}\text { Did not } \\
\text { reduce the } \\
\text { risk of falling }\end{array}$ & $\begin{array}{c}\text { Sage Journals } \\
\text { - Clinical } \\
\text { Rehabilitation }\end{array}$ \\
\hline 7 & $\begin{array}{c}\text { Daly et al. } \\
\text { [15] }\end{array}$ & $\begin{array}{l}\text { Effectiveness of } \\
\text { dual-task functional } \\
\text { power training for } \\
\text { preventing falls } \\
\text { in older people: } \\
\text { study protocol for a } \\
\text { cluster randomised } \\
\text { controlled trial }\end{array}$ & $>64$ & TUG & $\begin{array}{l}\text { Strength } \\
\text { exercises }\end{array}$ & 2 & $\begin{array}{l}\text { moderate- } \\
\text { to high- } \\
\text { intensity } \\
\text { 45-60 min }\end{array}$ & 18 & $\begin{array}{l}\text { Reduced the } \\
\text { risk of falling }\end{array}$ & $\begin{array}{c}\text { BMC } \\
\text { Research }\end{array}$ \\
\hline
\end{tabular}




\begin{tabular}{|c|c|c|c|c|c|c|c|c|c|c|}
\hline 8 & $\begin{array}{l}\text { Bohrer et } \\
\text { al. [16] }\end{array}$ & $\begin{array}{l}\text { Multicomponent } \\
\text { training program } \\
\text { with high-speed } \\
\text { movement execution } \\
\text { of ankle muscles } \\
\text { reduce risk of falls in } \\
\text { older adults }\end{array}$ & $>69$ & TUG & $\begin{array}{l}\text { Combination } \\
\text { of balance } \\
\text { and strength } \\
\text { exercises }\end{array}$ & 3 & $\begin{array}{l}\text { Low- to } \\
\text { moderate- } \\
\text { intensity } \\
45 \text { min }\end{array}$ & 3 & $\begin{array}{l}\text { Reduced the } \\
\text { risk of falling }\end{array}$ & $\begin{array}{l}\text { Rejuvenation } \\
\text { Reserarch }\end{array}$ \\
\hline 9 & $\begin{array}{l}\text { Pirauá et } \\
\text { al. [17] }\end{array}$ & $\begin{array}{c}\text { Effect of } 24 \text { week } \\
\text { strength training on } \\
\text { unstable surfaces on } \\
\text { mobility, balance, and } \\
\text { concern about falling } \\
\text { in older adults }\end{array}$ & $>68$ & TUG & $\begin{array}{l}\text { Combination } \\
\text { of balance } \\
\text { and strength } \\
\text { exercises }\end{array}$ & 3 & $\begin{array}{l}\text { Moderate } \\
\text { intensity } \\
60 \mathrm{~min}\end{array}$ & 3 & $\begin{array}{l}\text { Reduced the } \\
\text { risk of falling }\end{array}$ & $\begin{array}{l}\text { Scandinavian } \\
\text { Journal of } \\
\text { medicine e } \\
\text { Science in } \\
\text { Sports }\end{array}$ \\
\hline 10 & $\begin{array}{l}\text { Alfieri et al. } \\
\text { [18] }\end{array}$ & $\begin{array}{l}\text { Functional mobility } \\
\text { and balance in } \\
\text { community- dwelling } \\
\text { elderly submitted to } \\
\text { multisensory versus } \\
\text { strength exercises }\end{array}$ & $60-75$ & TUG & $\begin{array}{c}\text { Balance and } \\
\text { strength } \\
\text { exercises } \\
\text { separately }\end{array}$ & 2 & $\begin{array}{l}\text { moderate- } \\
\text { to high- } \\
\text { intensity } \\
60 \mathrm{~min}\end{array}$ & 3 & $\begin{array}{l}\text { Reduced the } \\
\text { risk of falling }\end{array}$ & $\begin{array}{l}\text { Clin Interv } \\
\text { Aging }\end{array}$ \\
\hline 11 & $\begin{array}{l}\text { Liu- } \\
\text { Ambrose et } \\
\text { al. [19] }\end{array}$ & $\begin{array}{l}\text { Otago Home-Based } \\
\text { Strength and Balance } \\
\text { Retraining Improves } \\
\text { Executive Functioning } \\
\text { in Older Fallers: } \\
\text { A Randomized } \\
\text { Controlled Trial }\end{array}$ & $>70$ & TUG & $\begin{array}{l}\text { Combination } \\
\text { of balance } \\
\text { and strength } \\
\text { exercises }\end{array}$ & - & $\begin{array}{l}\text { Low- to } \\
\text { moderate- } \\
\text { intensity } \\
60 \mathrm{~min}\end{array}$ & 12 & $\begin{array}{l}\text { Reduced the } \\
\text { risk of falling }\end{array}$ & $\begin{array}{l}\text { Journal of } \\
\text { the American } \\
\text { geriatrics } \\
\text { society }\end{array}$ \\
\hline 12 & $\begin{array}{c}\text { Yamada et } \\
\text { al. [20] }\end{array}$ & $\begin{array}{l}\text { Effect of resistance } \\
\text { training on physical } \\
\text { performance and fear } \\
\text { of falling in elderly } \\
\text { with different levels } \\
\text { of physical well-being }\end{array}$ & $>65$ & TUG & $\begin{array}{l}\text { Strength } \\
\text { exercises }\end{array}$ & 2 & $\begin{array}{l}\text { moderate- } \\
\text { to high- } \\
\text { intensity } \\
60 \text { min }\end{array}$ & 50 & $\begin{array}{l}\text { Reduced the } \\
\text { risk of falling }\end{array}$ & $\begin{array}{c}\text { Oxford Journal } \\
\text { - Age and } \\
\text { Ageing }\end{array}$ \\
\hline 13 & $\begin{array}{l}\text { Aartolahti } \\
\text { et al. [21] }\end{array}$ & $\begin{array}{l}\text { Long-term strength } \\
\text { and balance training } \\
\text { in prevention of } \\
\text { decline in muscle } \\
\text { strength and mobility } \\
\text { in older adults }\end{array}$ & $>76$ & TUG & $\begin{array}{l}\text { Combination } \\
\text { of balance } \\
\text { and strength } \\
\text { exercises }\end{array}$ & 1 & $\begin{array}{c}\text { Moderate } \\
\text { intensity } \\
75 \mathrm{~min}\end{array}$ & 27 & $\begin{array}{l}\text { Reduced the } \\
\text { risk of falling }\end{array}$ & $\begin{array}{c}\text { Aging } \\
\text { Clinical and } \\
\text { Experimental } \\
\text { Research }\end{array}$ \\
\hline 14 & $\begin{array}{c}\text { Lacroix et } \\
\text { al. [22] }\end{array}$ & $\begin{array}{c}\text { Effects of a } \\
\text { Supervised versus } \\
\text { an Unsupervised } \\
\text { Combined Balance } \\
\text { and Strength Training } \\
\text { Program on Balance } \\
\text { and Muscle Power in } \\
\text { Healthy Older Adults: } \\
\text { A Randomized }\end{array}$ & $65-80$ & TUG & $\begin{array}{l}\text { Combination } \\
\text { of balance } \\
\text { and strength } \\
\text { exercises }\end{array}$ & 3 & $\begin{array}{l}\text { moderate- } \\
\text { to high- } \\
\text { intensity } \\
60 \text { min }\end{array}$ & 3 & $\begin{array}{l}\text { Reduced the } \\
\text { risk of falling }\end{array}$ & Gerontology \\
\hline & & Controlled Trial & & & & & & & & \\
\hline 15 & $\begin{array}{c}\text { Miko et al. } \\
\text { [23] }\end{array}$ & $\begin{array}{l}\text { Effectiveness of } \\
\text { balance training } \\
\text { programme in } \\
\text { reducing the } \\
\text { frequency of falling } \\
\text { in established } \\
\text { osteoporotic women: } \\
\text { A randomized } \\
\text { controlled trial }\end{array}$ & $>65$ & $\begin{array}{l}\text { Berg Balance } \\
\text { Scale }\end{array}$ & $\begin{array}{c}\text { Balance } \\
\text { exercises }\end{array}$ & 3 & $\begin{array}{l}\text { Low- to } \\
\text { moderate- } \\
\text { intensity } \\
30 \text { min }\end{array}$ & 56 & $\begin{array}{l}\text { Reduced the } \\
\text { risk of falling }\end{array}$ & $\begin{array}{l}\text { Sage Journals } \\
\text { - Clinical } \\
\text { Rehabilitation }\end{array}$ \\
\hline 16 & $\begin{array}{c}\text { Joshua et } \\
\text { al. [24] }\end{array}$ & $\begin{array}{c}\text { Effectiveness } \\
\text { of Progressive } \\
\text { Resistance } \\
\text { Strength Training } \\
\text { Versus Traditional } \\
\text { Balance Exercise in } \\
\text { Improving Balance } \\
\text { Among the Elderly } \\
\text { - A Randomised } \\
\text { Controlled Trial }\end{array}$ & $>65$ & $\begin{array}{l}\text { Functional } \\
\text { reach test }\end{array}$ & $\begin{array}{l}\text { Balance, } \\
\text { strength and } \\
\text { combination } \\
\text { of balance } \\
\text { and strength } \\
\text { exercises }\end{array}$ & 4 & $\begin{array}{l}\text { Low- to } \\
\text { moderate- } \\
\text { intensity } \\
30 \text { min }\end{array}$ & 24 & $\begin{array}{l}\text { Reduced the } \\
\text { risk of falling }\end{array}$ & $\begin{array}{l}\text { Journal of } \\
\text { Clinical \& } \\
\text { Diagnostic } \\
\text { Research }\end{array}$ \\
\hline
\end{tabular}




\begin{tabular}{|c|c|c|c|c|c|c|c|c|c|c|}
\hline 17 & $\begin{array}{c}\text { Motalebi et } \\
\text { al. [25] }\end{array}$ & $\begin{array}{l}\text { Effect of low-cost } \\
\text { resistance training } \\
\text { on lower-limb } \\
\text { strength and balance } \\
\text { in institutionalized } \\
\text { seniors }\end{array}$ & $>64$ & TUG & $\begin{array}{l}\text { Strength } \\
\text { exercises }\end{array}$ & 2 & $\begin{array}{l}\text { Low- to } \\
\text { moderate- } \\
\text { intensity } \\
50 \text { min }\end{array}$ & 3 & $\begin{array}{l}\text { Reduced the } \\
\text { risk of falling }\end{array}$ & $\begin{array}{c}\text { Experimental } \\
\text { Aging } \\
\text { Research }\end{array}$ \\
\hline 18 & $\begin{array}{c}\text { Marques et } \\
\text { al. [26] }\end{array}$ & $\begin{array}{c}\text { Multicomponent } \\
\text { Training Program } \\
\text { with Weight-Bearing } \\
\text { Exercises Elicits } \\
\text { Favorable Bone } \\
\text { Density, Muscle } \\
\text { Strength, and Balance } \\
\text { Adaptations in Older } \\
\text { Women }\end{array}$ & $>68$ & TUG & $\begin{array}{l}\text { Balance and } \\
\text { strength } \\
\text { exercises } \\
\text { separately }\end{array}$ & 2 & $\begin{array}{l}\text { Low- to } \\
\text { moderate- } \\
\text { intensity } \\
60 \text { min }\end{array}$ & 32 & $\begin{array}{l}\text { Reduced the } \\
\text { risk of falling }\end{array}$ & $\begin{array}{c}\text { Calcified } \\
\text { Tissue } \\
\text { International }\end{array}$ \\
\hline 19 & $\begin{array}{l}\text { Lee et al. } \\
\text { [27] }\end{array}$ & $\begin{array}{l}\text { Balance Improvement } \\
\text { by Strength Training } \\
\text { for the Elderly }\end{array}$ & $>69$ & $\begin{array}{l}\text { Posturografia } \\
\text { estática } \\
\text { BioRescue }\end{array}$ & $\begin{array}{l}\text { Strength } \\
\text { exercises }\end{array}$ & 2 & $\begin{array}{l}\text { moderate- } \\
\text { to high- } \\
\text { intensity } \\
60 \text { min }\end{array}$ & 12 & $\begin{array}{l}\text { Reduced the } \\
\text { risk of falling }\end{array}$ & $\begin{array}{l}\text { Journal of } \\
\text { Physical } \\
\text { Therapy }\end{array}$ \\
\hline 20 & $\begin{array}{l}\text { Seco et al. } \\
\text { [28] }\end{array}$ & $\begin{array}{c}\text { A long-term } \\
\text { physical activity } \\
\text { training program } \\
\text { increases strength } \\
\text { and flexibility and } \\
\text { improves balance in } \\
\text { older adults. }\end{array}$ & $>65$ & $\begin{array}{c}\text { Balance } \\
\text { Evaluation } \\
\text { Systems Test }\end{array}$ & $\begin{array}{l}\text { Combination } \\
\text { of aerobic } \\
\text { exercises, } \\
\text { balance, and } \\
\text { strength } \\
\text { exercises }\end{array}$ & 3 & $\begin{array}{l}\text { Low- to } \\
\text { moderate- } \\
\text { intensity } \\
50-55 \text { min }\end{array}$ & 9 & $\begin{array}{l}\text { Reduced the } \\
\text { risk of falling }\end{array}$ & $\begin{array}{l}\text { Rehabilitation } \\
\text { Nursing } \\
\text { Journal }\end{array}$ \\
\hline 21 & $\begin{array}{l}\text { Gschwind } \\
\text { et al. [29] }\end{array}$ & $\begin{array}{l}\text { A best practice } \\
\text { falls prevention } \\
\text { exercise program } \\
\text { to improve balance, } \\
\text { strength/ power, } \\
\text { and psychosocial } \\
\text { health in older } \\
\text { adults: study protocol } \\
\text { for a randomized } \\
\text { controlled trial }\end{array}$ & $65-80$ & TUG & $\begin{array}{l}\text { Combination } \\
\text { of balance } \\
\text { and strength } \\
\text { exercises }\end{array}$ & 3 & $\begin{array}{l}\text { Low- to } \\
\text { moderate- } \\
\text { intensity } \\
30 \text { min }\end{array}$ & 3 & $\begin{array}{l}\text { Reduced the } \\
\text { risk of falling }\end{array}$ & $\begin{array}{c}\text { BMC } \\
\text { Geriatrics }\end{array}$ \\
\hline 22 & $\begin{array}{c}\text { LaStayo et } \\
\text { al. [30] }\end{array}$ & $\begin{array}{l}\text { Eccentric versus } \\
\text { traditional resistance } \\
\text { exercise for older } \\
\text { adult fallers in } \\
\text { the community: a } \\
\text { randomized trial } \\
\text { within a multi- } \\
\text { component fall } \\
\text { reduction program }\end{array}$ & $>65$ & $6 \mathrm{MW}$ & $\begin{array}{l}\text { Strength } \\
\text { exercises }\end{array}$ & 3 & $\begin{array}{l}\text { moderate- } \\
\text { to high- } \\
\text { intensity } \\
60 \text { min }\end{array}$ & 3 & $\begin{array}{l}\text { Reduced the } \\
\text { risk of falling }\end{array}$ & BMC Geriatrics \\
\hline 23 & $\begin{array}{c}\text { Halvarsson } \\
\text { et al. [31] }\end{array}$ & $\begin{array}{l}\text { Long-term effects } \\
\text { of new progressive } \\
\text { group balance } \\
\text { training for elderly } \\
\text { people with } \\
\text { increased risk of } \\
\text { falling - a randomized } \\
\text { controlled trial }\end{array}$ & $>80$ & $\begin{array}{l}\text { Falls Efficacy } \\
\quad \text { Scale- } \\
\text { Internacional }\end{array}$ & $\begin{array}{c}\text { Balance } \\
\text { exercises }\end{array}$ & 3 & $\begin{array}{l}\text { Low- to } \\
\text { moderate- } \\
\text { intensity } \\
60 \text { min }\end{array}$ & 12 & $\begin{array}{l}\text { Reduced the } \\
\text { risk of falling }\end{array}$ & $\begin{array}{l}\text { Sage Journals } \\
\text { - Clinical } \\
\text { Rehabilitation }\end{array}$ \\
\hline 24 & $\begin{array}{l}\text { Jacobson et } \\
\text { al. [32] }\end{array}$ & $\begin{array}{l}\text { Independent static } \\
\text { balance training } \\
\text { contributes to } \\
\text { increased stability } \\
\text { and functional } \\
\text { capacity in } \\
\text { community-dwelling } \\
\text { elderly people: } \\
\text { a randomized } \\
\text { controlled trial }\end{array}$ & $>80$ & $\begin{array}{l}\text { Berg Balance } \\
\text { Scale }\end{array}$ & $\begin{array}{c}\text { Balance } \\
\text { exercises }\end{array}$ & 3 & $\begin{array}{l}\text { Low- to } \\
\text { moderate- } \\
\text { intensity } \\
60 \text { min }\end{array}$ & 12 & $\begin{array}{l}\text { Reduced the } \\
\text { risk of falling }\end{array}$ & $\begin{array}{l}\text { Sage Journals } \\
\text { - Clinical } \\
\text { Rehabilitation }\end{array}$ \\
\hline 25 & $\begin{array}{l}\text { Sparrow et } \\
\text { al. [33] }\end{array}$ & $\begin{array}{l}\text { Increases in Muscle } \\
\text { Strength and Balance } \\
\text { Using a Resistance } \\
\text { Training Program } \\
\text { Administered Via a } \\
\text { Telecommunications } \\
\text { System in Older } \\
\text { Adults }\end{array}$ & $50-94$ & $6 \mathrm{MW}$ & $\begin{array}{l}\text { Strength } \\
\text { exercises }\end{array}$ & 3 & $\begin{array}{l}\text { moderate- } \\
\text { to high- } \\
\text { intensity } \\
60\end{array}$ & 12 & $\begin{array}{l}\text { Reduced the } \\
\text { risk of falling }\end{array}$ & $\begin{array}{l}\text { Oxford } \\
\text { Journals }\end{array}$ \\
\hline
\end{tabular}




\section{Quality Assessment of Studies}

The studies evaluated by the PEDro scale presented an average score of 8.6 points. One study scored 6 points [12], seven studies scored 7 points $[4,11,16,27,28,31,32]$, eight studies scored 8 points $[9,10,19,21,23,24,29,30]$, one study scored 9 points [25], and eight studies scored 10 points $[7,15,17,18,20,22,26,33]$ (Table 2).

Table 2: Assessment Study Quality Using the PEDro Scale for Randomized Clinical Trials.

\begin{tabular}{|c|c|c|c|c|c|c|c|c|c|c|c|c|}
\hline Items & $\begin{array}{l}\text { Eligibili- } \\
\text { ty Crite- } \\
\text { ria }\end{array}$ & $\begin{array}{c}\text { Random } \\
\text { Alloca- } \\
\text { tion }\end{array}$ & $\begin{array}{l}\text { Con- } \\
\text { cealed } \\
\text { Alloca- } \\
\text { tion }\end{array}$ & $\begin{array}{c}\text { Similar } \\
\text { at } \\
\text { Baseline }\end{array}$ & $\begin{array}{l}\text { Blinding } \\
\text { Subjects }\end{array}$ & $\begin{array}{c}\text { Blinding } \\
\text { Thera- } \\
\text { pists }\end{array}$ & $\begin{array}{c}\text { Blinding } \\
\text { Asses- } \\
\text { sors }\end{array}$ & $\begin{array}{l}\text { Ade- } \\
\text { quate } \\
\text { Fol- } \\
\text { low-up }\end{array}$ & $\begin{array}{c}\text { Inten- } \\
\text { tion to } \\
\text { Treat } \\
\text { Analysis }\end{array}$ & $\begin{array}{c}\text { Between- } \\
\text { Group } \\
\text { Statistical } \\
\text { Comparison }\end{array}$ & $\begin{array}{l}\text { Point Es- } \\
\text { timate/ } \\
\text { Measure } \\
\text { of Vari- } \\
\text { ability }\end{array}$ & $\begin{array}{l}\text { Score } \\
\text { Total }\end{array}$ \\
\hline $\begin{array}{c}\text { Teixeira et } \\
\text { al. [4] }\end{array}$ & $\mathrm{Y}$ & $\mathrm{Y}$ & & $\mathrm{Y}$ & $\mathrm{Y}$ & & & $\mathrm{Y}$ & $\mathrm{Y}$ & $\mathrm{Y}$ & Y & 07-Oct \\
\hline $\begin{array}{c}\text { Sousa et al. } \\
{[7]}\end{array}$ & $\mathrm{Y}$ & $\mathrm{Y}$ & Y & Y & Y & $\mathrm{Y}$ & Y & $\mathrm{Y}$ & $\mathrm{Y}$ & $\mathrm{Y}$ & Y & $10-0 c t$ \\
\hline $\begin{array}{c}\text { Cancela et } \\
\text { al. [9] }\end{array}$ & $\mathrm{Y}$ & $\mathrm{Y}$ & & $\mathrm{Y}$ & $\mathrm{Y}$ & & $\mathrm{Y}$ & $\mathrm{Y}$ & $\mathrm{Y}$ & $\mathrm{Y}$ & $\mathrm{Y}$ & 08-Oct \\
\hline $\begin{array}{c}\text { Ansai et al. } \\
{[10]}\end{array}$ & $\mathrm{Y}$ & $\mathrm{Y}$ & & Y & $\mathrm{Y}$ & & $\mathrm{Y}$ & $\mathrm{Y}$ & $\mathrm{Y}$ & $\mathrm{Y}$ & Y & 08-0ct \\
\hline $\begin{array}{l}\text { Hiyamizu } \\
\text { et al. [11] }\end{array}$ & $\mathrm{Y}$ & $\mathrm{Y}$ & Y & Y & $\mathrm{Y}$ & & & & $\mathrm{Y}$ & $\mathrm{Y}$ & $\mathrm{Y}$ & 07-Oct \\
\hline $\begin{array}{c}\text { Varela et } \\
\text { al. [12] }\end{array}$ & $\mathrm{Y}$ & & & $\mathrm{Y}$ & $\mathrm{Y}$ & & & $\mathrm{Y}$ & $\mathrm{Y}$ & Y & Y & 06-Oct \\
\hline $\begin{array}{c}\text { Daly et al. } \\
\text { [15] }\end{array}$ & $\mathrm{Y}$ & $\mathrm{Y}$ & Y & Y & $\mathrm{Y}$ & $\mathrm{Y}$ & $\mathrm{Y}$ & $\mathrm{Y}$ & $\mathrm{Y}$ & $\mathrm{Y}$ & Y & $10-0 c t$ \\
\hline $\begin{array}{c}\text { Bohrer et } \\
\text { al. [16] }\end{array}$ & $\mathrm{Y}$ & $\mathrm{Y}$ & & Y & $\mathrm{Y}$ & & $\mathrm{Y}$ & & $\mathrm{Y}$ & $\mathrm{Y}$ & Y & 07-Oct \\
\hline $\begin{array}{l}\text { Pirauá et } \\
\text { al. [17] }\end{array}$ & $\mathrm{Y}$ & $\mathrm{Y}$ & Y & $\mathrm{Y}$ & $\mathrm{Y}$ & Y & $\mathrm{Y}$ & $\mathrm{Y}$ & $\mathrm{Y}$ & $\mathrm{Y}$ & $\mathrm{Y}$ & 10-Oct \\
\hline $\begin{array}{l}\text { Alfieri et } \\
\text { al. [18] }\end{array}$ & $\mathrm{Y}$ & $\mathrm{Y}$ & Y & $\mathrm{Y}$ & $\mathrm{Y}$ & $\mathrm{Y}$ & Y & $\mathrm{Y}$ & $\mathrm{Y}$ & $\mathrm{Y}$ & Y & 10-Oct \\
\hline $\begin{array}{c}\text { Liu- } \\
\text { Ambrose } \\
\text { et al. [19] }\end{array}$ & $\mathrm{Y}$ & $\mathrm{Y}$ & & Y & $\mathrm{Y}$ & & $\mathrm{Y}$ & $\mathrm{Y}$ & $\mathrm{Y}$ & $\mathrm{Y}$ & $\mathrm{Y}$ & 08-Oct \\
\hline $\begin{array}{c}\text { Yamada et } \\
\text { al. [20] }\end{array}$ & $\mathrm{Y}$ & $\mathrm{Y}$ & Y & Y & $\mathrm{Y}$ & $\mathrm{Y}$ & $\mathrm{Y}$ & Y & $\mathrm{Y}$ & $\mathrm{Y}$ & Y & 10-0ct \\
\hline $\begin{array}{l}\text { Aartolahti } \\
\text { et al. [21] }\end{array}$ & $\mathrm{Y}$ & $\mathrm{Y}$ & & $\mathrm{Y}$ & $\mathrm{Y}$ & & Y & Y & $\mathrm{Y}$ & $\mathrm{Y}$ & Y & 08-Oct \\
\hline $\begin{array}{c}\text { Lacroix et } \\
\text { al. [22] }\end{array}$ & $\mathrm{Y}$ & $\mathrm{Y}$ & $\mathrm{Y}$ & $\mathrm{Y}$ & $\mathrm{Y}$ & Y & $\mathrm{Y}$ & $\mathrm{Y}$ & $\mathrm{Y}$ & $\mathrm{Y}$ & $\mathrm{Y}$ & 10-Oct \\
\hline $\begin{array}{c}\text { Miko et al. } \\
\text { [23] }\end{array}$ & $\mathrm{Y}$ & $\mathrm{Y}$ & Y & Y & $\mathrm{Y}$ & & & $\mathrm{Y}$ & $\mathrm{Y}$ & $\mathrm{Y}$ & Y & 08-Oct \\
\hline $\begin{array}{c}\text { Joshua et } \\
\text { al. [24] }\end{array}$ & Y & $\mathrm{Y}$ & Y & $\mathrm{Y}$ & Y & & & Y & $\mathrm{Y}$ & $\mathrm{Y}$ & $\mathrm{Y}$ & 08-Oct \\
\hline $\begin{array}{l}\text { Motalebi } \\
\text { et al. [25] }\end{array}$ & Y & Y & $\mathrm{Y}$ & $\mathrm{Y}$ & $\mathrm{Y}$ & Y & $\mathrm{Y}$ & & $\mathrm{Y}$ & $\mathrm{Y}$ & Y & 09-0ct \\
\hline $\begin{array}{c}\text { Marques et } \\
\text { al. [26] }\end{array}$ & $\mathrm{Y}$ & $\mathrm{Y}$ & Y & $\mathrm{Y}$ & $\mathrm{Y}$ & Y & $\mathrm{Y}$ & $\mathrm{Y}$ & $\mathrm{Y}$ & $\mathrm{Y}$ & $\mathrm{Y}$ & $10-0 c t$ \\
\hline $\begin{array}{c}\text { Lee et al. } \\
\text { [27] }\end{array}$ & $\mathrm{Y}$ & $\mathrm{Y}$ & Y & & $\mathrm{Y}$ & & & $\mathrm{Y}$ & $\mathrm{Y}$ & $\mathrm{Y}$ & Y & 07-Oct \\
\hline $\begin{array}{c}\text { Seco et al. } \\
\text { [28] }\end{array}$ & $\mathrm{Y}$ & Y & & Y & $\mathrm{Y}$ & & $\mathrm{Y}$ & & $\mathrm{Y}$ & $\mathrm{Y}$ & $\mathrm{Y}$ & 07-Oct \\
\hline $\begin{array}{l}\text { Gschwind } \\
\text { et al. [29] }\end{array}$ & $\mathrm{Y}$ & Y & & $\mathrm{Y}$ & $\mathrm{Y}$ & & $\mathrm{y}$ & $\mathrm{Y}$ & $\mathrm{Y}$ & $\mathrm{Y}$ & $\mathrm{Y}$ & 08-0ct \\
\hline $\begin{array}{l}\text { LaStayo et } \\
\text { al. [30] }\end{array}$ & $\mathrm{Y}$ & $\mathrm{Y}$ & & $\mathrm{Y}$ & $\mathrm{Y}$ & & $\mathrm{Y}$ & $\mathrm{Y}$ & $\mathrm{Y}$ & $\mathrm{Y}$ & $\mathrm{Y}$ & 08-Oct \\
\hline $\begin{array}{c}\text { Halvarsson } \\
\text { et al. [31] }\end{array}$ & Y & $\mathrm{Y}$ & Y & Y & & & & $\mathrm{Y}$ & $\mathrm{Y}$ & $\mathrm{Y}$ & Y & 07-Oct \\
\hline
\end{tabular}




\begin{tabular}{|c|c|c|c|c|c|c|c|c|c|c|c|c|}
\hline $\begin{array}{l}\text { Jacobson } \\
\text { et al. [32] }\end{array}$ & $\mathrm{Y}$ & $\mathrm{Y}$ & Y & Y & $\mathrm{Y}$ & & & & Y & $\mathrm{Y}$ & Y & 07-Oct \\
\hline $\begin{array}{c}\text { Sparrow et } \\
\text { al. [33] }\end{array}$ & $\mathrm{Y}$ & $\mathrm{Y}$ & $\mathrm{Y}$ & Y & Y & $\mathrm{Y}$ & Y & Y & Y & Y & Y & 10-Oct \\
\hline
\end{tabular}

Y: Contemplated item.

*Item 1 does not contribute to the total score.

\section{Discussion}

This systematic review analyzed the effects of training programs on the risk of falling in older adults. We mainly aimed to elucidate whether the risk of falling is positively affected by isolated or combined training programs, and thus contribute to the prevention of falls and reduction in the risk of mortality. Regular exercise influenced total daily physical activity and possibly induced chronic physiological adaptations. We observed that all studies that combined strength training programs with balance exercises reduced the risk of falling [4,16-19,21,22,24,26,29]. The same was seen in isolated strength training programs [15,20,24,25,27,30,33]. According to studies by Wolfson, et al. [34], Lord, et al. [35], and Howe, et al. [36], the findings of this review can in part be attributed to improvements in neuromuscular coordination by and "training specificity" of the programs applied in the studies cited here. However, positive effects on muscle mass, strength, composition, power, and torque production, as well as changes in the mechanical properties of the tendon, must be observed to explain the significant reduction in the risk of falling $[37,38]$. Balance training, as well as the evaluation of this variable, is considered an essential component of fall prevention programs [39].

The loss of balance is directly related to decreases in muscle mass, strength, and proprioception. According to Teixeira, et al. [4], a strength training program for the quadriceps combined with proprioception training can decrease the risk of falling in postmenopausal women, in addition to increasing strength and static and dynamic balance. Therefore, proprioception and strength exercises are of great importance in reducing the risk of falling in older adults. Sousa, et al. [7] found a decrease in the risk of falling after the aerobic and balance training program. The risk of falling also decreases when the strength protocol integrates aerobic and balance training [28]. However, Cancela, et al. [9] observed no decrease in the risk of falling after a strength, aerobic, and balance training program. Ansai, et al. [10], who used an aerobic and strength training program, likewise found no decrease. Both studies used samples of older adults over 80 years old. This age group has less organic adaptation, and the studies justified, in a respective way, the differences attributable to the short period of intention, application of light physical activity in a sitting position, and failure to monitor falls daily.

No reduction in the risk of falling was also found in Varela, et al. [12], who used isolated aerobic training in older adults with mild cognitive impairment. According to $\mathrm{Xu}$, et al. [40], aerobic activities can improve balance and generates a positive effect on the proprioception of older adults, given that aerobic exercises decrease gait instability and can decrease degenerative processes in the nervous and muscular systems. As such, aerobic exercises can contribute to decreasing the risk of falls in older adults [41]. In studies that used isolated balance exercises, Jacobson, et al.[32], Halvarsson, et al. [31], and Miko, et al. [23] reported a decrease in the risk of falling. However, these results were not observed in Hiyamizu, et al. [11]. A plausible explanation regarding the divergent results is the lack of non-standardized protocols in the experiments. According to Thomas, et al. [42], training programs that offer an approach that includes resistance exercises, aerobic components, and proprioception work can improve balance and reduce the risk of falling in older adults. Falls are multifactorial events that may be related to intrinsic and extrinsic factors. The incidence of falls increases each year in the older population worldwide, with a positive relation between age and the increase in cases of falls.

Older adults can have several complications after a fall, such as fractures and head injuries; indeed, falls account for $11 \%$ of the mortality rate in this population [43-45]. Thus, increased mortality in older adults is related to physical inactivity [46]. Moreover, Thomas, et al. [42] reported that low-intensity exercises improve the balance of older adults and contribute to decreasing the risk of falling; adherence to moderate- to high-intensity training programs is less attractive, leading to lower adherence, especially in older adults. Based on the studies above, and in recognition of the importance and relevance of reducing falling risk for maintaining the quality of life and decreasing falls-related mortality in the older population, we propose that strength and aerobic training programs with proprioception exercises be recommended when and included in designing interventions. The strengths of this systematic review are that it was based on reliable results and full-text studies and it included high-quality papers. Meanwhile, the main limitation of this study is the lack of standardized assessment instruments for the risk of falling. Nonetheless, the instruments were validated and reliable, and thus, we chose to include all instruments.

\section{Conclusion}

This systematic review analyzed the effects of training programs on the risk of falls in older adults. Combined or multicomponent training programs, as well as isolated strength training, could decrease the risk of falling in older people. Further studies should standardize the protocols of training programs and assessment 
instruments, to enable further analysis of the aspects that brings better benefits: whether it is the training program, the modality to be worked on, or the modulation of the variables in the training.

\section{Implications for Practice}

The findings, based on the studies analyzed, demonstrated the importance and relevance of physical training programs for decreasing the risk of falling in people and older adults. Therefore, strength and aerobic training programs are recommended, along with balance exercises, for older adults.

\section{Author Contributions}

Silva A designed the research; Andrade G, Nascimento J, Ribeiro A and Barbosa C performed the research; Silva A, Silva J and Oliveira J analyzed the data; Silva A, Oliveira J wrote the paper; Silva A, Pertille A, Silva J and Oliveira J supervised the paper; all authors read and approved the final manuscript.

\section{PRISMA 2009 Checklist}

The authors have read the PRISMA 2009 Checklist, and the manuscript was prepared and revised according to the PRISMA 2009 Checklist.

\section{Acknowledgments}

The authors would like to thank the Coordenação de Aperfeiçoamento de Pessoal de Nível Superior-Brasil (CAPES) for the financial support through scholarships-Finance Code 001.

\section{References}

1 (2018) Insituto Brasileiro de Geografia e Estatisticas (IBGE) - Estatísticas Sociais. Em 2018, expectativa de vida era de 76,3 anos. Rio de Janeiro: Agência IBGE Notícias.

2 Matsudo SM, Matsudo VKR, Barros Neto TL (2001) Atividade física e envelhecimento: aspectos epidemiológicos. Rev Bras Med Esporte 7(1): 2-13.

3 Fujita K, Kaburagi H, Nimura A, Miyamoto T, Wakabayashi Y, et al. (2019) Lower grip strength and dynamic body balance in women with distal radial fractures. Osteoporos Int 30(5): 949-956.

4 Teixeira LE, Silva KN, Imoto AM, Teixeira TJ, Kayo AH, et al. (2010) Progressive load training for the quadriceps muscle associated with proprioception exercises for the prevention of falls in postmenopausal women with osteoporosis: a randomized controlled trial. Osteoporos Int 21(4): 589-596.

5 Oliveira RJd, Lima RM, Gentil P, Simões HG, Ávila WRdM, et al. (2008) Respostas hormonais agudas a diferentes intensidades de exercícios resistidos em mulheres idosas. Rev Bras Med Esporte 14(4): 367-371.

6 Gasparotto LPR, Falsarella GR, Coimbra AMV (2014) As quedas no cenário da velhice: conceitos básicos e atualidades da pesquisa em saúde. Rev Bras Geriatr Gerontol 17(1): 201-209.

7 Sousa N, Mendes R, Silva A, Oliveira J (2017) Combined exercise is more effective than aerobic exercise in the improvement of fall risk factors: a randomized controlled trial in community-dwelling older men. Clin Rehabil 31(4): 478-486.

8 Zhao R, Zhao M, Xu Z (2015) The effects of differing resistance training modes on the preservation of bone mineral density in postmenopausal women: a meta-analysis. Osteoporos Int 26(5): 1605-1618.
9 Cancela Carral JM, Pallin E, Orbegozo A, Ayan Perez C (2017) Effects of Three Different Chair-Based Exercise Programs on People Older Than 80 Years. Rejuvenation Res 20(5): 411-419.

10 Ansai JH, Aurichio TR, Goncalves R, Rebelatto JR (2016) Effects of two physical exercise protocols on physical performance related to falls in the oldest old: A randomized controlled trial. Geriatr Gerontol Int 16(4): 492-499.

11 Hiyamizu M, Morioka S, Shomoto K, Shimada T (2012) Effects of dual task balance training on dual task performance in elderly people: a randomized controlled trial. Clin Rehabil 26(1): 58-67.

12 Varela S, Ayan C, Cancela JM, Martin V (2012) Effects of two different intensities of aerobic exercise on elderly people with mild cognitive impairment: a randomized pilot study. Clin Rehabil 26(5): 442-450.

13 Moher D, Liberati A, Tetzlaff J, Altman DG, Group P (2009) Preferred reporting items for systematic reviews and meta-analyses: the PRISMA statement. PLoS Med 6(7): e1000097.

14 Maher CG, Sherrington C, Herbert RD, Moseley AM, Elkins M (2003) Reliability of the PEDro scale for rating quality of randomized controlled trials. Phys Ther 83(8): 713-721.

15 Daly RM, Duckham RL, Tait JL, Rantalainen T, Nowson CA, et al. (2015) Effectiveness of dual-task functional power training for preventing falls in older people: study protocol for a cluster randomised controlled trial. Trials 16: 120.

16 Bohrer RCD, Pereira G, Beck JK, Lodovico A, Rodacki ALF (2019) Multicomponent Training Program with High-Speed Movement Execution of Ankle Muscles Reduces Risk of Falls in Older Adults. Rejuvenation Res 22(1): 43-50.

17 Piraua ALT, Cavalcante BR, de Oliveira VMA, Beltrao NB, de Amorim Batista G, et al. (2019) Effect of 24-week strength training on unstable surfaces on mobility, balance, and concern about falling in older adults. Scand J Med Sci Sports 29(11): 1805-1812.

18 Alfieri FM, Riberto M, Gatz LS, Ribeiro CP, Lopes JA, et al. (2010) Functional mobility and balance in community-dwelling elderly submitted to multisensory versus strength exercises. Clin Interv Aging 5: 181-185.

19 Liu Ambrose T, Donaldson MG, Ahamed Y, Graf P, Cook WL, et al. (2008) Otago home-based strength and balance retraining improves executive functioning in older fallers: a randomized controlled trial. J Am Geriatr Soc 56(10): 1821-1830.

20 Yamada M, Arai H, Uemura K, Mori S, Nagai K, et al. (2011) Effect of resistance training on physical performance and fear of falling in elderly with different levels of physical well-being. Age Ageing 40(5): 637-641.

21 Aartolahti E, Lonnroos E, Hartikainen S, Hakkinen A (2020) Long-term strength and balance training in prevention of decline in muscle strength and mobility in older adults. Aging Clin Exp Res 32(1): 59-66.

22 Lacroix A, Kressig RW, Muehlbauer T, Gschwind YJ, Pfenninger B, et al. (2016) Effects of a Supervised versus an Unsupervised Combined Balance and Strength Training Program on Balance and Muscle Power in Healthy Older Adults: A Randomized Controlled Trial. Gerontology 62(3): 275-288.

23 Miko I, Szerb I, Szerb A, Poor G (2017) Effectiveness of balance training programme in reducing the frequency of falling in established osteoporotic women: a randomized controlled trial. Clin Rehabil 31(2): 217-224.

24 Joshua AM, D'Souza V, Unnikrishnan B, Mithra P, Kamath A, et al. (2014) Effectiveness of progressive resistance strength training versus traditional balance exercise in improving balance among the elderly - a randomised controlled trial. J Clin Diagn Res 8(3): 98-102.

25 Motalebi SA, Cheong LS, Iranagh JA, Mohammadi F (2018) Effect of low-cost resistance training on lower-limb strength and balance in institutionalized seniors. Exp Aging Res 44(1): 48-61. 
26 Marques EA, Mota J, Machado L, Sousa F, Coelho M, et al. (2011) Multicomponent training program with weight-bearing exercises elicits favorable bone density, muscle strength, and balance adaptations in older women. Calcif Tissue Int 88(2): 117-129.

27 Lee IH, Park SY (2013) Balance improvement by strength training for the elderly. J Phys Ther Sci 25(12): 1591-1593.

28 Seco J, Abecia LC, Echevarria E, Barbero I, Torres Unda J, et al. (2013) A long-term physical activity training program increases strength and flexibility and improves balance in older adults. Rehabil Nurs 38(1): 3747.

29 Gschwind YJ, Kressig RW, Lacroix A, Muehlbauer T, Pfenninger B, et al. (2013) A best practice fall prevention exercise program to improve balance, strength / power, and psychosocial health in older adults: study protocol for a randomized controlled trial. BMC Geriatr 2013; 13: 105.

30 LaStayo P, Marcus R, Dibble L, Wong B, Pepper G (2017) Eccentric versus traditional resistance exercise for older adult fallers in the community: a randomized trial within a multi-component fall reduction program. BMC Geriatr 2017; 17(1): 149

31 Halvarsson A, Franzen E, Faren E, Olsson E, Oddsson L, et al. (2013) Long-term effects of new progressive group balance training for elderly people with increased risk of falling - a randomized controlled trial. Clin Rehabil 27(5): 450-458.

32 Jacobson BH, Thompson B, Wallace T, Brown L, Rial C (2011) Independent static balance training contributes to increased stability and functiona capacity in community-dwelling elderly people: a randomized controlled trial. Clin Rehabil 25(6): 549-556.

33 Sparrow D, Gottlieb DJ, Demolles D, Fielding RA (2011) Increases in muscle strength and balance using a resistance training program administered via a telecommunications system in older adults. J Gerontol A Biol Sci Med Sci 66(11): 1251-1257.

34 Wolfson L, Whipple R, Derby C, Judge J, King M, et al. (1996) Balance and strength training in older adults: intervention gains and Tai Chi maintenance. J Am Geriatr Soc 44(5): 498-506.

35 Lord SR, Sherrington C, Menz HB (2001) Epidemiology of falls and fall-related injuries. In: Lord SR, Sherrington C, Menz HB. Falls in

ISSN: 2574-1241

DOI: 10.26717/BJSTR.2021.34.005564

José Jonas de Oliveira. Biomed J Sci \& Tech Res

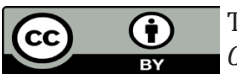

This work is licensed under Creative Commons Attribution 4.0 License

Submission Link: https://biomedres.us/submit-manuscript.php older people: Risk factors and strategies for prevention. Cambridge: Cambridge Univeristy Press, p. 3-16.

36 Howe TE, Rochester L, Neil F, Skelton DA, Ballinger C (2011) Exercise for improving balance in older people. Cochrane Database Syst Rev 2011(11): CD004963

37 Daubney ME, Culham EG (1999) Lower-extremity muscle force and balance performance in adults aged 65 years and older. Phys Ther 79(12): 1177-1185.

38 Lang T, Streeper T, Cawthon P, Baldwin K, Taaffe DR, et al. (2010) Sarcopenia: etiology, clinical consequences, intervention, and assessment. Osteoporos Int 21(4): 543-559.

39 Hakim RM, DiCicco J, Burke J, Hoy T, Roberts E (2004) Differences in balance related measures among older adults participating in Tai Chi, structured exercise, or no exercise. J Geriatr Phys Ther, p. 13-17.

40 Xu D, Hong Y, Li J, Chan K (2004) Effect of tai chi exercise on proprioception of ankle and knee joints in old people. Br J Sports Med 38(1): 50-54.

41 Rubenstein LZ (2006) Falls in older people: epidemiology, risk factors and strategies for prevention. Age Ageing 35 Suppl 2: ii37-ii41.

42 Thomas E, Battaglia G, Patti A, Brusa J, Leonardi V, et al. (2019) Physical activity programs for balance and fall prevention in elderly: A systematic review. Medicine (Baltimore) 98(27): e16218.

43 Pitchai P, Dedhia HB, Bhandari N, Krishnan D, D’Souza NRJ, et al. (2019) Prevalence, risk factors, circumstances for falls and level of functional independence among geriatric population - A descriptive study. Indian J Public Health 63(1): 21-26.

44 Kumar A, Srivastava D, Verma A, Kumar S, Singh N, et al. (2013) The problems of fall, risk factors and their management among geriatric population in India. Indian J Community Health 25(2): 89-94.

45 Dsouza SA, Rajashekar B, Dsouza HS, Kumar KB (2014) Falls in Indian older adults: a barrier to active ageing. Asian J Gerontol Geriatr 9(1): 33-40.

46 Simonsick EM, Lafferty ME, Phillips CL, Mendes de Leon CF, Kasl SV, et al. (1993) Risk due to inactivity in physically capable older adults. Am J Public Health 83(10): 1443-1450.

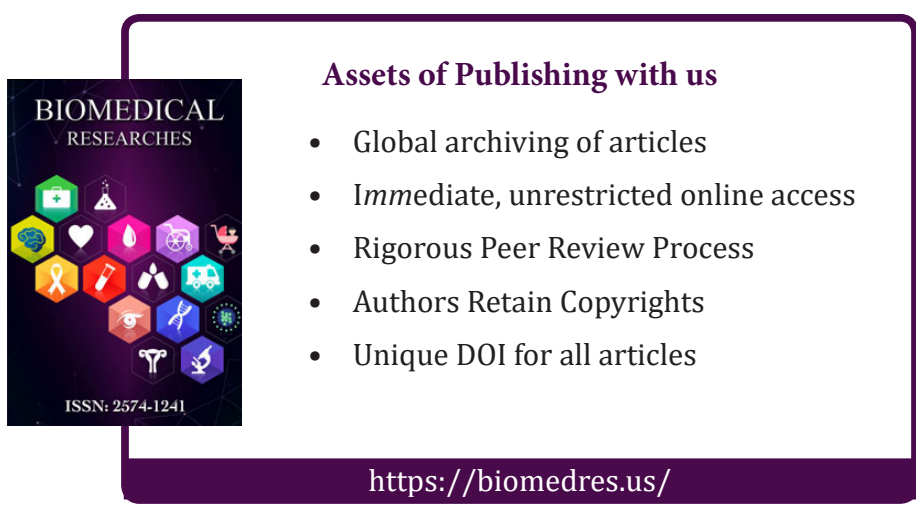

Copyright@ José Jonas de Oliveira | Biomed J Sci \& Tech Res | BJSTR. MS.ID.005564. 